\title{
Análise bibliométrica da produção científica brasileira sobre Dipteryx odorata no período de 2009 a 2018
}

A Dipteryx odorata é uma espécie nativa da Amazônia e conhecida popularmente como cumaru. Sua madeira é de alta densidade e é bastante utilizada na construção civil e naval. O fruto do cumaru apresenta uma semente de onde se extrai o óleo da cumarina. O óleo é utilizado como fixador de essências e é largamente utilizada com este propósito na indústria de cosméticos. O presente trabalho realizou uma revisão bibliométrica sobre a espécie Dipteryx odorata nos últimos 10 anos (2009 a 2018) com a finalidade de avaliar a ênfase e dinâmica de publicações a respeito da espécie. Para a pesquisa foram usadas as seguintes palavras-chaves: 'Dipteryx odorata, cumaru e cumaru-ferro', onde posteriormente foi aplicado a filtragem de busca por anos e por país obtendo-se uma amostragem de 60 publicações que citavam alguma das palavras-chaves. Como principais resultados verificou-se que houve um aumento de publicações com o passar dos anos no período de estudo. A maior quantidade de artigos que citavam o cumaru se encaixava na área de conhecimento de silvicultura e manejo. 0 Pará é o estado que lidera com o número de publicações sobre o cumaru enquanto a Embrapa é a instituição de pesquisa que mais publicou a respeito da espécie.

Palavras-chave: Cumaru; Bibliometria; Cumarina; Amazônia.

\section{Bibliometric analysis of brazilian scientific production on Dipteryx odorata from 2009 to 2018}

Dipteryx odorata is a native Amazonian species and popularly known as cumaru. Its wood is high density and is widely used in construction and shipbuilding. The fruit of the cumaru has a seed from which the coumarin oil is extracted. The oil is used as an essence fixer and is widely used for this purpose in the cosmetics industry. The present work carried out a bibliometric review about the species Dipteryx odorata in the last 10 years (2009 to 2018) in order to evaluate the emphasis and dynamics of publications regarding the species. For the research, the following keywords were used: 'Dipteryx odorata, cumaru and cumaru-ferro', where the search filtering by years and by country was subsequently applied, obtaining a sample of 60 publications that cited any of the keywords. As main results it was found that there was an increase of publications over the years in the study period. Most articles citing cumaru fit into the area of knowledge of forestry and management. Pará is the state that leads with the number of publications on cumaru, while Embrapa is the most published research institution on the species.

Keywords: Cumaru; Bibliometrics; Coumarin; Amazon.

Topic: Ciências Florestais

Reviewed anonymously in the process of blind peer
Received: 04/12/2019

Approved: 11/01/2020
João Gabriel Almeida Portela

Universidade Federal do Oeste do Pará, Brasi

http://lattes.cnpq.br/002961793903785

http://orcid.org/0000-0002-7276-4152

joaoportelagabriel@gmail.com

Daniela Pauletto (iD

Universidade Federal do Oeste do Pará, Brasil

http://lattes.cnpq.br/0963317170667125

http://orcid.org/0000-0003-1855-6077

paulettoflorestal@gmail.com
Referencing this:

PORTELA, J. G. A.; PAULETTO, D.. Análise bibliométrica da produção científica brasileira sobre Dipteryx odorata no período de 2009 a 2018. Revista Ibero Americana de Ciências Ambientais, v.11, n.1, p.19-28, 2020. DOI: http://doi.org/10.6008/CBPC2179-6858.2020.001.0003 


\section{INTRODUÇÃO}

As florestas sempre estiveram presentes na vida da humanidade, tanto no fornecimento de produtos, como nos serviços ambientais (MOREIRA, 2011), e a Amazônia tem se destacado como uma grande fonte desses produtos, com destaque para os de origem florestais não madeireiros, com alto potencial econômico e socioambiental, oriundo em sua maior parte do extrativismo, economia que se baseia na exploração e conservação dos recursos naturais (RÊGO et al., 2017).

Esses produtos exercerem grande papel na renda de trabalhadores ou extrativistas, ao fornecerem segurança alimentar, matéria prima para indústria, além de ser utilizados para tratamentos de doenças (RÊGO et al., 2016). O extrativismo aliado ao manejo sustentável de madeira através de florestas plantadas ou sistemas agroflorestais com impacto reduzido podem proporcionar melhores retorno econômico do que outras atividades econômicas de uso da terra.

Dentre as espécies florestais típicas da floresta amazônica, que se destacam pelo seu potencial de mercado, temos a Dipteryx odorata conhecida como cumaru ou cumaru ferro. Nativa da América do Sul, o cumaru é uma espécie leguminosa, do tipo arbórea encontrada em todos os estados da Amazônia Legal (YANAI, 2012). Espécie pertence à família Fabaceae tem distribuição na região tropical (SILVA et al., 2010) sendo adaptada a florestas de terra firme e a várzea com arvores que podem alcançar $35 \mathrm{~m}$ de altura e 60 cm de diâmetro (YANAl, 2012).

Devido ao fato da espécie possuir estratégias de fácil adaptação, alta taxa de crescimento e elevada taxa de sobrevivência, permite rápida frutificação, além das vantagens de sintetizar o nitrogênio direto da atmosfera e transformar em alimentos (FÁBIO et al., 2018) tem se tornado uma cultura de grande valor a ser usada para reflorestamento. Além disso, o extrativismo desta matéria prima representa mais uma atividade remunerada para as famílias extrativistas do norte do Brasil (RÊGO et al., 2016), além de proporcionar um melhor bem-estar social a diversas famílias que podem adquirir novas opções de bens e serviços através dessa renda (SILVA et al., 2018).

Segundo Silva et al. (2010) o cumaru é utilizado em indústrias produtoras de óleos essenciais, perfumes, cosméticos, medicamentos, alimentos, fumo e bebidas, pelas suas propriedades aromáticas e terapêuticas. Abrangendo o mercado nacional e internacional, incluindo feiras, estabelecimentos locais e mercados mais especializados somente no estado do Pará, sua extração atinge $87,4 \%$ de toda a produção vegetal no país, sendo o estado que mais produz a amêndoa (IBGE, 2014). Além disso, apresenta madeira nobre, apresentando alta resistência estrutural e alta densidade, tornando-se amplamente utilizada na indústria madeireira (ZAU et al., 2014).

As amêndoas dos frutos são aromáticas, de onde se extrai um óleo essencial, a cumarina, de grande importância na economia regional. Esta substância é utilizada para fazer perfumes, cosméticos, bebidas, cigarros, anti-inflamatórios, herbicidas, fungicidas, além do seu uso medicinal o que justifica a crescente demanda pela substância no mercado nacional e internacional (PINTO et al., 2008). Ressalta-se, no entanto, que devido a produção sintética da cumarina a partir de outras plantas, houve redução da demanda da 
semente in natura para esses fins (RÊGO et al., 2016), demonstrando a necessidade de condução de plantios de cumaru também para a extração de madeira.

Segundo Homma (2014) é grande o potencial extrativo da semente de cumaru, necessitando da organização de comunidades, beneficiamento e comercialização. Ainda não há domínio total desse potencial presente na biodiversidade nativa, podendo futuramente aliar preservação ambiental, renda e qualidade de vida para os agricultores da Amazônia.

Para ampliar a difusão de conhecimentos acerca do cumaru, suas propriedades e benefícios é necessário conhecer as áreas do conhecimento que mais publicam e como está vem sendo realizada no Brasil. Para estes indicadores uma das técnicas utilizadas é a bibliometria, técnica quantitativa e estatística empregada para medir índices de produção e disseminação do conhecimento, bem como acompanhar o desenvolvimento de diversas áreas cientificas e os padrões de autoria, publicação e uso dos resultados de investigação (ARAúJO, 2015).

Segundo Santos (2015) já é de conhecimento em diversas áreas, estudos que analisam produções científicas. Essas análises são importantes uma vez que se tornam indispensáveis a fundamentação de ideias para que pesquisadores possam se atentar as diferentes formas que o conhecimento vem sendo gerado em seu campo de estudo, onde se destacam as pesquisas bibliométricas como forma de analisar esses estudos.

Esses estudos permitem conhecer as tradições, os paradigmas, assim como os documentos e formas de expressão e influências mútuas em relação aos diferentes campos e domínios científicos, permitindo uma perspectiva mais profunda e coerente quando se busca entender os documentos, a organização, os sistemas, a informação e o conhecimento. Assim, com o intuito de promover o enriquecimento do conhecimento científico a respeito da pesquisa com Dipteryx odorata este estudo tem por objetivo realizar uma avaliação bibliometria da produção científica em revistas brasileiras nos períodos de 2009 a 2018.

\section{METODOLOGIA}

Este trabalho tem caráter descritivo, empregando a bibliometria como principal técnica de análise metodológica. Segundo Guedes et al. (2005) as principais leis bibliométricos são: Lei de Bradford, (produtividade de periódicos), Lei de Lotka (produtividade científica de autores) e Leis de Zipf (frequência de palavras) e tem como produto a Ciência da informação objetivando criar informações que possuem utilidade ou que podem ser uteis no futuro.

Essa técnica, também descrita por Araújo (2006), como de caráter quantitativo e estatístico, se propõe à medição dos níveis de produção e disseminação do conhecimento científico, empregando técnicas estatísticas. Como método de análise foram escolhidos artigos publicados em revistas científicas brasileiras, que têm por objetivo promover discussões, disseminar ideias e divulgar resultados de pesquisas (com enfoques locais, nacionais e internacionais) em temas florestais e agrícolas, devidamente indexadas e reconhecidas nacionalmente.

Para a seleção dos artigos foi utilizado o mecanismo de busca por filtros disponibilizado pela interface de pesquisa do site periódico CAPES. Além disso, foi utilizado o portal Google Acadêmico para acesso aos 
periódicos. As palavras-chaves utilizadas para o processo de pesquisa foram: Dipteryx Odorata, cumaru e cumaru-ferro. Posteriormente, foi realizada a leitura de cada artigo, para triagem e classificação das principais informações, fornecendo uma base de dados confiante para a realização desta pesquisa. Utilizouse o software Microsoft Office Excel 2013 para tabulação dos dados das publicações do período de 2009 a 2018 em revistas indexadas no portal periódicos.capes.org.br e disponíveis no Google Acadêmico.

\section{DISCUSSÃO TEÓRICA}

Através da análise dos periódicos, houve a necessidade de classificá-los conforme as áreas de estudo de cada publicação. Segundo a CAPES, classificar as áreas do conhecimento tem como finalidade eminentemente prática, objetivando proporcionar às instituições de ensino, pesquisa e inovação uma maneira ágil e funcional de sistematizar e prestar informações concernentes a projetos de pesquisa e recursos humanos aos órgãos gestores da área de ciência e tecnologia. Este trabalho identificou algumas das áreas do conhecimento com base na classificação da CAPES e portal de pesquisa Google Acadêmico

A pesquisa resultou em uma amostragem de 60 publicações de diversas áreas temáticas. Baseado nas áreas do conhecimento, separou-se as publicações (Figura 1) onde se destaca a Silvicultura e Manejo que detém maior número de artigos relacionados ao cumaru. Estas áreas envolvem publicações que retratam os sistemas agroflorestais, os quintais agroflorestais, diferentes tipos de plantios (homogêneo, nativo e consorciado) e retrata também as práticas de manejo em geral.

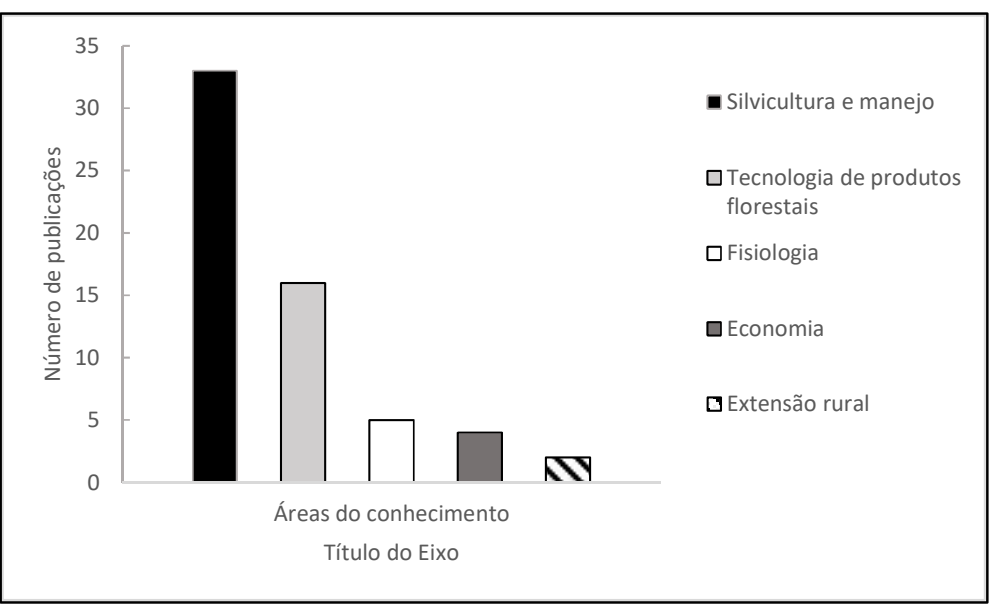

Figura 1: Número de publicações divididos por áreas do conhecimento da produção científica brasileira sobre a Dipteryx odorata no período de 2009 a 2018.

A tecnologia de produtos florestais ficou em segundo lugar no número de publicações, o que mostra que há bastante interesse nos produtos extraídos desta árvore que são o óleo essencial da semente (cumarina) e a madeira que possui alto valor de mercado. Seguindo pela grande área da Fisiologia Vegetal e da área de Economia e Extensão Rural representando 82\% e 3\% das publicações, respectivamente.

Foram identificadas poucas publicações do tema de Economia que é uma área de extrema importância para dimensionar o valor dos produtos e subprodutos do cumaru no cenário mundial e como está sendo comercializada esse produto nativo da Amazônia. A obtenção de dados sobre a economia da espécie implica em dificuldades na aquisição de informações confiáveis pois muitos produtores não têm a 
rotina de controle de custos e produção em seu dia-a-dia. A tabela 1 mostra a quantidade de publicações (\%), por tema secundário abordado na publicação científica.

Tabela 1: Número de publicações (\%) divididos por temas secundários da produção científica brasileira sobre a Dipteryx odorata no período de 2009 a 2018.

\begin{tabular}{|l|c|}
\hline \multicolumn{1}{|c|}{ Temas secundários } & $\begin{array}{c}\text { No de } \\
\text { Publicações (\%) }\end{array}$ \\
\hline Sistemas agroflorestais e quintais agroflorestais & 23,2 \\
\hline Tecnologia da madeira & 14,2 \\
\hline Plano de manejo e exploração madeireira & 14,2 \\
\hline Uso e mercado de sementes (PFNM) & 12,5 \\
\hline Crescimento em plantios & 9,0 \\
\hline Extração e química de Cumarina & 7,2 \\
\hline Conhecimento tradicional sobre D. odorata & 5,4 \\
\hline Fenologia e Biologia Reprodutiva & 5,4 \\
\hline Crescimento de mudas & 5,4 \\
\hline Recuperação de áreas degradadas & 3,5 \\
\hline
\end{tabular}

Dentre os temas secundários, existem os sistemas agroflorestais (SAFs) incluindo também os quintais agroflorestais (QAFs), que possuem a maior quantidade de publicações $(21,7 \%)$ e, por isso, se mostra como tema de maior importância. Segundo Almeida et al. (2014) quintal agroflorestal (QAF) é um sistema tradicional de uso da terra amplamente empregado nas regiões tropicais. Os quintais agroflorestais são muito presentes na Amazônia como forma de produção e diversificação alimentar e se constituem como importantes espaços de provisão e recreação. Além disso, o cumaru tem sido uma espécie promissora e amplamente utilizada em sistemas agroflorestais pela precocidade de produção de amêndoas.

Os Sistemas agroflorestais são uma estratégia de recuperação dos ecossistemas danificados pela agropecuária, sendo fonte de renda para o pequeno e grande produtor rural. Desta forma, estudos relacionados a melhoria da qualidade do solo, inclusão de espécies florestais em áreas degradadas podem contribuir na melhor utilização de áreas a serem exploradas economicamente, demonstrando a importância dos SAFs como meio de subsistência, de promoção da segurança alimentar e de otimização do uso da terra (ALMEIDA et al., 2014). O cumaru é uma espécie florestal bastante presente na produção agrária familiar.

Nos SAFs é possível combinar os benefícios da produção de alimentos, forragem, energia, madeira, óleos e outros, com os serviços de conservação do solo, manutenção da fertilidade, ciclagem de nutrientes, controle de erosão, fixação de nitrogênio e uma maior estabilidade do microclima na propriedade agrícola. (RAYOL et al., 2019).

Em seguida vem os temas de tecnologia da madeira $(13,3 \%)$ e plano de manejo e exploração madeireira (13,3\%). Estes resultados devem estar relacionados ao potencial madeireiro da cultura, uma vez que sua madeira é tida como uma das melhores devido sua durabilidade e por não se fender quando exposta ao sol. É utilizada também em implementos agrícolas, construção naval, cabos de ferramentas, moirões, carroçaria, estacas, esteios, eixo de moinhos, tacos para soalho o que torna de alto interesse para estudos relacionados com tecnologia e exploração madeireira.

Além disso, pesquisas sobre tecnologia da madeira e manejo florestal devem apresentar novos resultados para diversificar as opções de madeira e evitar o exagero no uso de determinadas espécies encontradas em menor quantidade na floresta. $\mathrm{O}$ uso de madeira menos nobre reduz o perigo de extinção 
das espécies que são atualmente mais procuradas na floresta Amazônica, além de favorecer o manejo florestal, pois com o aumento do número de espécies potenciais, é possível aumentar a produção por unidade de área e adotar opções de espécies para colheita (ADEODATO et al., 2011).

O manejo florestal é a forma de garantir o uso dos recursos racionalmente, a manutenção da biodiversidade e sustentar a indústria madeireira que, ainda, é um dos maiores geradores de renda da região norte. Ainda nesse sentido, esses estudos com essa temática são importantes para compreender como as espécies se comportam, ajudando na tomada de decisão sobre como conduzir a floresta.

Além disso, estudos sobre as propriedades físicas e anatômicas de uma madeira são importantes pois auxiliam no processo final para a escolha de um melhor uso dela. Outro fator a ser considerado é a densidade, que se torna um dos mais importante uma vez que se relaciona com a estrutura anatômica e suas composições químicas, o que inclui também resistência, dimensão e qualidade da superfície (SOARES et al. 2017).

Estudos com uso e mercado das sementes apresentou 11,7\% dos trabalhos pesquisados, seguido crescimento de plantios com $(8,4 \%)$, extração e química de cumarina $(6,7 \%)$, conhecimento tradicional sobre cumaru, fenologia e biologia reprodutiva e crescimento de mudas $5 \%$ cada e recuperação de áreas degradas com $3,3 \%$.

Os estudos fenológicos e biologia reprodutiva, que no trabalho apresentou (5\%), são importantes para silvicultura, manejo florestal e ecologia, pois analisam o desenvolvimento das plantas quanto aos seus eventos vegetativos e reprodutivos no decorrer de um período, bem como das relações desses eventos com fatores ambientais e bióticos. Esses estudos são de extrema importância, uma vez que, a partir deles, muitos outros estudos envolvendo biologia reprodutiva, coleta de frutos e sementes, e dispersão de diásporos podem ser desenvolvidos (SOUZA et al., 2014).

Portanto, a fenologia estuda a frequência, a intensidade, além do sincronismo de eventos denominados de fenofases, como o brotamento e a queda de folhas, a floração e a frutificação, fornecendo dados que permitem a análise da dependência destas fenofases com fatores bióticos e abióticos. Entretanto, são raros os estudos fenológicos de longa duração em florestas tropicais com observação direta de plantas, não ultrapassando em geral 10 anos de observações (CHAPMAN et al., 2005; PINTO et al., 2008).

O crescimento de mudas representou 5\% dos trabalhos publicados sobre cumaru no período de 2009 a 2018, sendo uma das principais áreas para desenvolvimento de trabalho acerca do cumaru. Estudos relacionados com a produção de mudas e fatores que influenciam em seu desenvolvimento como densidade, substrato, água e luz são fundamentais para o desenvolvimento de tecnologias de produção de mudas com alto padrão de qualidade, o que torna necessários estudos acerca de produção de mudas florestais de cumaru. Mudas desenvolvidas em viveiro podem receber condições ótimas para seu desenvolvimento inicial, aumentando as suas chances de se estabelecerem no campo. Mudas com alto padrão de qualidade tem maior possibilidade de originarem plantios sadios e mais produtivos (FREITAS et al., 2014).

Ainda segundo Freitas et al. (2014) para as espécies amazônicas, existe pouca informação sobre as técnicas de viveiro que produzem mudas mais vigorosas. Com o aumento da demanda por essas informações, 
principalmente em decorrência do avanço do desmatamento e multiplicação de áreas degradadas, é de vital importância que estudos sejam realizados visando contribuir com informações sobre as espécies nativas.

Estudos sobre conhecimento tradicional no qual na presente pesquisa apresentou $5 \%$ dos trabalhos publicados é uma área de conhecimento importante para região amazônica, uma vez que, mesmo com os avanços de tendências globais, este conhecimento pode ser encontrado em povos de diferentes regiões, apresentando historias e tradições culturais únicas, que contribuem para que a ciência possa comprovar tais eficiências uma vez citados apenas a partir do conhecimento das pessoas. Além disso o uso de plantas valoriza a cultura dessas comunidades, além de contribuir com a conservação, recomposição e manutenção de áreas agricultáveis.

Ainda nesse sentido, plantios florestais para recuperação de áreas degradadas com espécies nativas, ainda são em número reduzido, por isso estudos com essa finalidade, são ferramentas importantes para nortear estratégias de reabilitação e recuperação das mesmas (LIMA JÚNIOR et al., 2009). O cumaru por apresentar rápido crescimento e ser uma espécie florestal que frutifica precocemente, aos 4 ou 5 anos de idade (CARVALHO, 2009), vem sendo bastante requisitada em plantios agroflorestais e de reflorestamentos. Para uma melhor compreensão dos dados, distribui-se a produção dos periódicos por estados, como mostra a Figura 2.

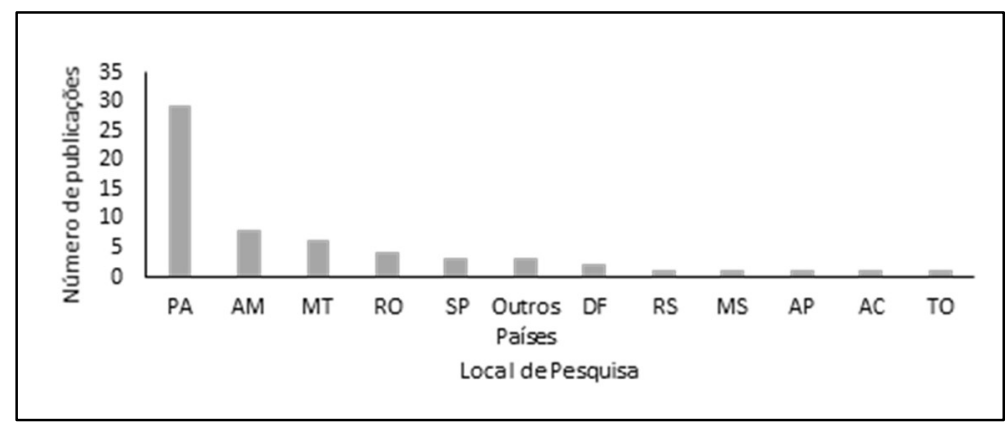

Figura 2: Número de publicações por local de origem (estados) da produção cientifica brasileira sobre Dipteryx odorata no período de 2009 a 2018.

No total de onze estados, Pará e Amazonas apresentaram 37 das 60 publicações, evidenciando que a pesquisa do cumaru está diretamente ligada ao seu local de ocorrência que também é nos estados do Pará e Amazonas. Apesar disso, também existem trabalhos de alta relevância nos demais estados o que mostra que a espécie Cumaru é altamente difundida em todo o Brasil. Além disso, foram encontrados trabalhos que produzidos por pesquisadores do exterior (Santa Cruz na Bolívia, Pardubice na Tchéquia, e Ucayali no Peru). Esses artigos foram produzidos por instituições estrangeiras, porém foram publicados em periódicos brasileiros o que permitiram o ingresso neste trabalho.

Devido ao seu grande potencial econômico e industrial, nos últimos anos a produção de cumaru aumentou no Brasil, com destaque de produção para os estados do Pará e no município de Alenquer (IBGE, 2014). Este aumento da demanda pela espécie para indústria tanto mercado nacional, como no internacional, deve ter sido um dos motivos para o aumento de pesquisas acerca do cumaru, destacando assim o estado do Pará como um dos estados com maiores produções cientificas ocasionado também pelo surgimento de novas universidades no interior da Amazônia o que gerou novos cursos na área de ciências agrárias. 
O Amazonas também se destaca na disseminação de conhecimentos acerca do cumaru, devido à presença de instituiç̧̃es de pesquisas como INPA e UFAM. Com isso, ele é por ser o segundo maior estado na produção cientifica do cumaru. A tabela 2 mostra as instituições de vínculo do primeiro autor em relação as publicações sobre cumaru ao longo do período estudado.

Tabela 2: Número de publicações por instituições que desenvolveram pesquisas sobre a Dipteryx odorata no período de 2009 a 2018.

\begin{tabular}{|c|c|}
\hline Instituição & $\begin{array}{c}\text { № de } \\
\text { Publicações }\end{array}$ \\
\hline Empresa Brasileira de Pesquisa Agropecuária (EMBRAPA) & 12 \\
\hline Universidade Federal do Oeste do Pará (UFOPA) & 9 \\
\hline Universidade Federal do Amazonas (UFAM) & 5 \\
\hline Universidade Federal do Mato Grosso (UFMT) & 4 \\
\hline Universidade Federal Rural da Amazônia (UFRA) & 3 \\
\hline Instituto Chico Mendes de Biodiversidade (ICMBio) & 3 \\
\hline Universidade Federal de Rondônia (UNIR) & 3 \\
\hline Serviço Florestal Brasileiro - (SFB) & 2 \\
\hline Instituto Nacional de Pesquisa da Amazônia (INPA) & 2 \\
\hline Museu Paraense Emílio Goeldi (MPEG) & 1 \\
\hline Parque Ecológico de Gunma (PEG) & 1 \\
\hline Centro de Ciências Exatas, da Natureza e de Tecnologia (CENT) & 1 \\
\hline Universidade Estadual Paulista (UNESP) & 1 \\
\hline Universidade Federal de Paraná (UFPR) & 1 \\
\hline Universidade Federal do Pampa (UNIPAMPA) & 1 \\
\hline Universidade Luterana do Brasil (ULBRA) & 1 \\
\hline Instituto Boliviano de Investigación Forestal (IBIF) & 1 \\
\hline Pontifícia Universidade Católica de Minas (PUC/Minas) & 1 \\
\hline Universidad Complutense de Madrid (UCM) & 1 \\
\hline Universidade Federal do Amapá (UFAP) & 1 \\
\hline Universidade Federal do Pará (UFPA) & 1 \\
\hline Universidade Federal do Mato Grosso do Sul (UFMS) & 1 \\
\hline University of Pardubice & 1 \\
\hline Instituto Federal do Amazonas (IFAM) & 1 \\
\hline Universidade Nacional de Ucayali (UNU) & 1 \\
\hline Universidade Federal de Roraima (UFRR) & 1 \\
\hline Total Geral & 60 \\
\hline
\end{tabular}

A Tabela 2, apresenta a Embrapa como principal instituição de pesquisa do cumaru, com 12 publicações. Seguido pela Universidade Federal do Oeste do Pará (UFOPA), com 9 publicações. Isso porque a EMBRAPA é referência em pesquisa com temática em Ciências agrárias, e contribui significativamente com a pesquisa científica em parceria com outras instituições.

A UFOPA, mostrou-se uma instituição de grande contribuição ao desenvolvimento de publicações do cumaru, mesmo sendo uma instituição com poucos anos de existência. Ao longo dos seus 10 anos, movimentou trabalhos de pesquisa cientifica e extensão na região, trabalhando no desenvolvimento da interdisciplinaridade entre as grandes áreas do conhecimento abordadas nesta pesquisa.

Os dados permitiram o desenvolvimento do gráfico a seguir, trazendo os anos com maiores publicações. O Gráfico 3 apresenta o número de publicações em cada ano desta pesquisa, mostrando que elas aumentaram com o passar do tempo, sendo os anos de 2017 e 2018 os anos com mais publicações. Isso pode ser visto de forma positiva porque mostra um aumento das pesquisas do cumaru. 


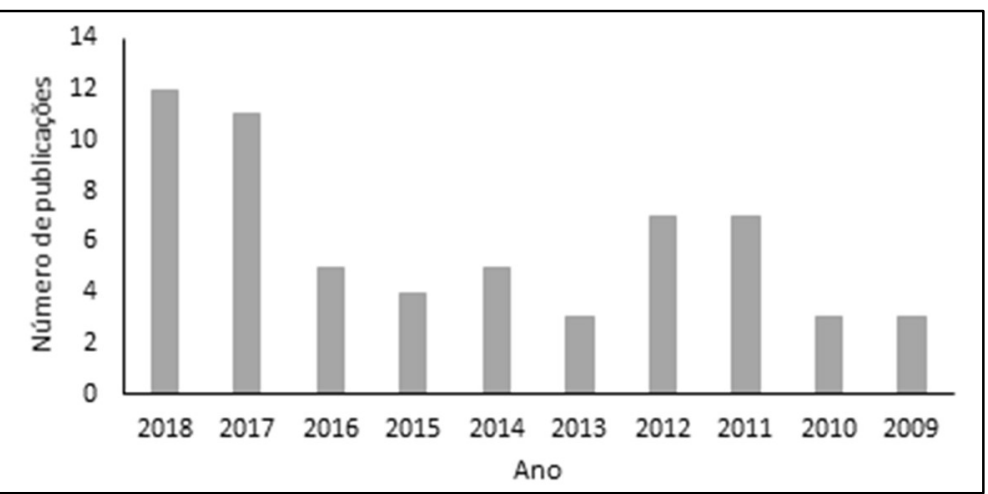

Gráfico 3: Número de publicações por ano da produção científica brasileira sobre a Dipteryx odorata no período de 2009 a 2018.

O interesse das organizações não governamentais, institutos de pesquisa, dentre outros, tem aumentado nos últimos tempos, proporcionando novas informações sobre a importância de produtos no contexto socioeconômico das populações, dentre eles o cumaru, além dos efeitos sobre a conservação e o manejo das florestas (RÊGO et al., 2016).

Este aumento de estudos sobre o cumaru nos últimos anos também pode estar relacionado ao seu uso para exploração de madeira e pelas indústrias que produzem óleos essenciais, perfumes, cosméticos, medicamentos, alimentos, fumo e bebidas, pelas suas propriedades aromáticas e terapêuticas, abrangendo o mercado nacional e internacional (SILVA et al., 2010). Ainda neste sentido existe uma carência de estudos que demonstre como ampliar a produção do cumaru de maneira economicamente viável e sustentável.

\section{CONCLUSÕES}

Com o levantamento bibliométrico da espécie Dipteryx odorata foi diagnosticado que a espécie é uma essência florestal muito valiosa, com bastante potencial de expansão e que ainda há poucas publicações especificas mostrando que o cumaru carece de estudos em todas as áreas do conhecimento. Contudo ainda sim houve um aumento de publicações ao passar dos anos no período de estudo. A maior quantidade de publicações que citaram o cumaru esteve inserida na área de conhecimento de silvicultura e manejo. O Pará é o estado que lidera com o número de publicações do cumaru e a Embrapa é a instituição de pesquisa que mais publicou a respeito do cumaru nos últimos dez anos.

\section{REFERÊNCIAS}

ADEODATO, S.; VILELA, M.; BETIOL, L. S.; MONZONI, M.. Madeira de ponta a ponta: o caminho desde a floresta até o consumo. São Paulo: RAE, 2011.

ALMEIDA, L. S.; GAMA, J. R. V.. Quintais agroflorestais: estrutura, composição florística e aspectos socioambientais em área de assentamento rural na Amazônia brasileira. Ciência florestal, Santa Maria, v.24, n.4, p.10371048, 2014. DOI: http://dx.doi.org/10.1590/1980$\underline{509820142404023}$

ARAÚJO, R. M. F.. Análise bibliométrica da produção científica sobre plantas alimentícias não convencionais (PANC) nos cursos de pós-graduação do Brasil. Monografia
(Graduação em Nutrição) - Universidade Federal do Rio Grande do Norte, Natal, 2015.

ARAÚJO. C. A.. Bibliometria: evolução histórica e questões atuais. Revista Em Questão, Porto Alegre, v.12, n.1, p.11-32, 2006.

CARVALHO, P. E. R.. Comunicado Técnico: Cumaru-Ferro Dipteryx odorata. 6 ed. Colombo: Embrapa Florestas, 2009.

CASTANHA, R. C. G.; GRÁCIO, M. C. C.. Análise estatística unimultivariada como método de avaliação de programas de pós-graduação: uma análise bibliométrica na área de filosofia (2010-2012). Brazilian Journal of Information 
Studies: Research Trends, v.10, n.3, p.54-68, 2016. DOI: http://doi.org/10.36311/1981-1640.2016.v10n3.07.p54

CHAPMAN, C. A.; CHAPMAN, L. J.; STRUHSAKEI, T. T.; ZANNE, A. E.; CLARK, C. J.; POULSEN, J. R. A.. long-term evaluation of fruiting phenology: importance of climate change. Journal of Tropical Ecology, v.21, n.1, p.31-45, 2005. DOI: https://doi.org/10.1017/S0266467404001993

FÁBIO, F. G.; REBELLATO, L.; CÂNCIO, I. A. P.. Manejo florestal do cumaru: um experimento rentável e sustentável em Óbidos, Estado do Pará. 2018. In: SEMINÁRIO INTERNACIONAL EM CIÊNCIAS DO AMBIENTE E SUSTENTABILIDADE NA AMAZÔNIA, 5. Anais. Manaus: UFAM, 2018.

FREITAS, A. F.; SOUZA, L. A. G.; CARDOSO, I. M.; PAIVA, H. N.. Fino de carvão vegetal em substrato para produção de mudas de Dipteryx odorata. Revista Brasileira de Agroecologia, v.9, n.3, p.31-40, 2014.

GUEDES, V. L. S.; BORSCHIVER, S.. Bibliometria: uma ferramenta estatística para a gestão da informação e do conhecimento, em sistemas de informação, de comunicação e de avaliação científica e tecnológica. In: ENCONTRO NACIONAL DE CIÊNCIA DA INFORMAÇÃO, 6. Anais. Salvador: UFBA, 2005.

HOMMA, A. K. O.. Embrapa Amazônia Oriental: Extrativismo vegetal na Amazônia: história, ecologia, economia e domesticação. 1 ed. Brasília: Embrapa, 2014.

IBGE. Instituto Brasileiro de Geografia e Estatística. Produção da extração vegetal e da silvicultura. Rio de Janeiro: IBGE, 2014.

LIMA JÚNIOR, J. F. M.; BARBOSA, A. P.; LOPES, R. B. C.. Análise do crescimento do cumaru (Dipteryx odorata Aubl. Willd) e jatobá (Hymenaea courbaril L. varo courbaril) em plantios puros e mistos em recuperação de áreas degradadas pela pecuária extensiva. In: JORNADA DE INICIAÇÃO CIENTÍFICA PIBIC CNPQ/FAPEAM/INPA, 18. Anais. Manaus: INPA, 2009.

MOREIRA, J. M. M. Á P.. Potencial e participação das florestas na matriz energética. Pesquisa Florestal Brasileira, Colombo, v.31, n.68, p.363-372, 2011. DOI: http://doi.org/10.4336/2011.pfb.31.68.363

PINTO, A. M.; MORELLATO, L. P. C.; BARBOSA, A. P.. Fenologia reprodutiva de Dipteryx odorata (Aubl.) Willd (Fabaceae) em duas áreas de floresta na Amazônia Central. Acta Amazônica, Manaus, v.38, n.4, p.643-650, 2008. DOI: http://dx.doi.org/10.1590/S0044-59672008000400006

RAYOL, B. P.; ALVINO-RAYOL, F. O.. Desenvolvimento inicial de espécies arbóreas em sistemas agroflorestais no Baixo Amazonas, Pará, Brasil. Revista de Ciências
Agroveterinárias, v.18, n.1, p.59-64, 2019.

DOI: http://doi.org/10.5965/223811711812019059

RÊGO, L. J. S.; SILVA, M. L.; SILVA, L. F.; GAMA, J. R. V.; REIS, L. P.. Comercialização da amêndoa de cumaru nos municípios de Santarém e Alenquer, leste da Amazônia.

Revista de Administração e Negócios da Amazônia, v.8, n.3, p.338-361, 2016. DOI: http://doi.org/10.18361/21768366/rara.v8n3p338-361

RÊGO, L. J. S.; SILVA, M. L.; SILVA, L. S.; GAMA, J. R. V.; REIS, L. P.; REIS, P. C.. Caracterização do consumo de amêndoa de cumaru na Amazônia Oriental. Biota Amazônia, Macapá, v.7, n.3, p.23-27, 2017. DOI: http://dx.doi.org/10.18561/21795746/biotaamazonia.v7n3p23-27

SANTOS, G. C.. Análise bibliométrica dos artigos publicados como estudos bibliométricos na história do congresso brasileiro de custos. Pensar Contábil, Rio de Janeiro, v.17, n.62, p.4-13, 2015.

SILVA, A. A., SANTOS, L. E.; CRUZ, G. S.; RIBEIRO, R. B. S.; GAMA, J. R. V.. Potencial de comercialização de produtos florestais não madeireiros na área de manejo da reserva extrativista Tapajós Arapiuns Pará. Acta tecnológica, São Luis, v.13, n.1, p.45-63, 2018.

SILVA, T. M. S.; JARDIM, F. C. S.; SILVA, M. S. P. S.. Mercado de amêndoas de Dipteryx odorada (cumaru) no estado do Pará. Floresta, Curitiba, v.40, n.3, p.603-614, 2010. DOI: http://dx.doi.org/10.5380/fr.v40i3.18922

SOARES, A. A.; TORRES, F. B.; PEREIRA, A. C. M.; COSTA, J. S.; SILVA, M. G.. Comparação anatômica e descrição da densidade e macroscopicidade da espécie Dipteryx odorata (Aubl.) Willd (cumaru). 2017. In: CONGRESO LATINOAMERICANO DE ESTRUCTURAS DA MADERA E II CONGRESO IBERO-LATINOAMERICANO DE LA MADERA EN LA CONSTRUCCIÓN, 2. Anais. Buenos Aires: UNNOBA, 2017.

SOUZA, D. N. N.; CAMACHO, R. G. V.; MELO, J. I. M.; ROCHA, L. N. G.; SILDA, N. F.. Estudo fenológico de espécies arbóreas nativas em uma unidade de conservação de caatinga no Estado do Rio Grande do Norte, Brasil. Biotemas, Florianópolis, v.27, n.2, p.31-42, 2014. DOI: http://dx.doi.org/10.5007/2175-7925.2014v27n2p31

YANAI, A. E.. Patentes de produtos naturais amazônicos: análise de impacto da inovação tecnológica mundial. Dissertação (Mestrado em Ciência, Tecnologia e Sociedade) Universidade Federal do São Carlos, São Carlos, 2012.

ZAU, M. D. L.; VASCONCELOS, R. P.; GIACON, V. M.; LAHR, F. A. R.. Avaliação das Propriedades Química, Física e Mecânica de Painéis Aglomerados Produzidos com Resíduo de Madeira da Amazônia-Cumaru (Dipteryx Odorata) e Resina Poliuretana à Base de Óleo de Mamona. Polímeros, São Carlos, v.24, n.6, p.726-732, 2014. DOI:

http://dx.doi.org/10.1590/0104-1428.1594

A CBPC - Companhia Brasileira de Produção Científica (CNPJ: 11.221.422/0001-03) detém os direitos materiais desta publicação. Os direitos referem-se à publicação do trabalho em qualquer parte do mundo, incluindo os direitos às renovações, expansões e disseminações da contribuição, bem como outros direitos subsidiários. Todos os trabalhos publicados eletronicamente poderão posteriormente ser publicados em coletâneas impressas sob coordenação da Sustenere Publishing, da Companhia Brasileira de Produção Científica e seus parceiros autorizados. Os (as) autores (as) preservam os direitos autorais, mas não têm permissão para a publicação da contribuição em outro meio, impresso ou digital, em português ou em tradução. 